\title{
Model for creep threshold stress in precipitation-strengthened alloys with coherent particles
}

\author{
Emmanuelle A. Marquis, David C. Dunand * \\ Department of Materials Science and Engineering, Northwestern University, MLSB 1123, 2225 N. Campus Drive, Evanston, \\ IL 60208-3108, USA
}

Received 18 May 2002; accepted 22 May 2002

\begin{abstract}
The general climb model for creep threshold stress for dislocations interacting with incoherent particles is modified for the case of coherent precipitates, by taking into account elastic interactions between matrix dislocations and particles due to particle/matrix stiffness and lattice mismatches. The model is in qualitative agreement with experimental data for the Al-Sc system.
\end{abstract}

(C) 2002 Acta Materialia Inc. Published by Elsevier Science Ltd. All rights reserved.

Keywords: Creep; Threshold stress; Precipitation strengthening; Aluminum alloys; Dislocation mobility

\section{Introduction}

Creep threshold stresses observed in precipitation- or dispersion-strengthened alloys are explained by mechanisms based on particle shearing, bypass by climb, or detachment, the latter mechanism being operative only for incoherent particles [1]. For the case of climb bypass, two climb processes are possible [1]. The local climb model assumes a sharp bend of the dislocation at the particle-matrix interface [2]; since this bend is expected to relax by diffusion, the general climb model considering an equilibrium dislocation configuration was developed and predicts smaller threshold stresses [3]. However, the effects of lat-

\footnotetext{
${ }^{*}$ Corresponding author. Tel.: +1-847-491-5370; fax: +1-847467-6573.

E-mail address: dunand@northwestern.edu (D.C. Dunand).
}

tice and stiffness mismatches between matrix and particles are not considered in these models and can be important in precipitation-strengthened alloys with coherent particles. The present paper expands the general climb model developed by Rösler and Arzt [3] to consider these effects applicable to coherent precipitates.

\section{Model}

We consider cylindrical particles of diameter and height equal to $2 r$ (Fig. 1), rather than the cubic shape employed in the original general climb model [3]. In this manner, the ramp angle, $\beta$, (defined by the glide plane and the plane tangent to the surface of the particle at the point where the dislocation contacts the particle) is defined by geometrical considerations rather than being arbitrarily chosen. The cylindrical shape is a reasonable approximation of the spherical shape of the 


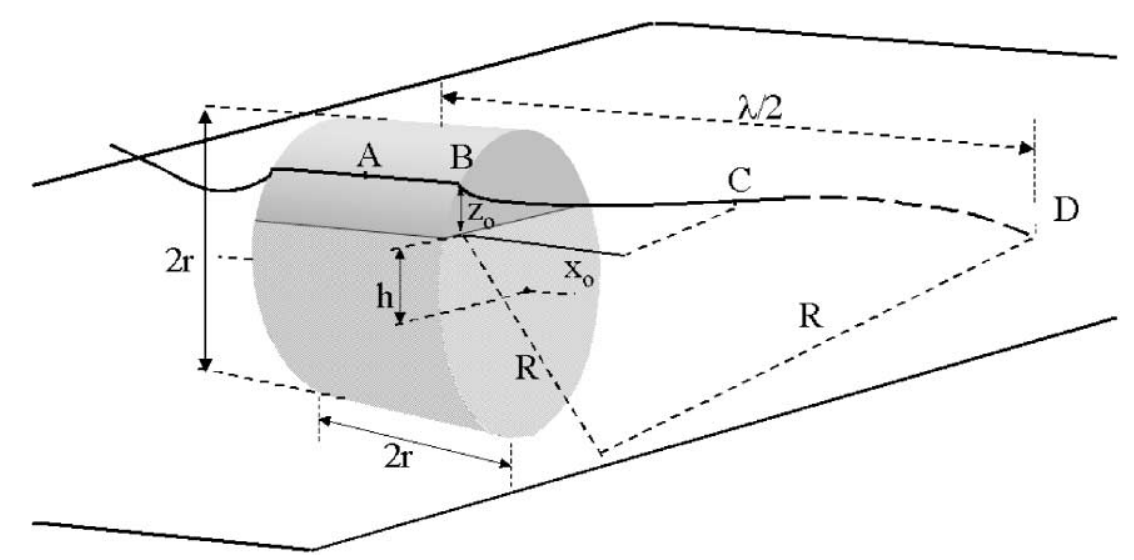

Fig. 1. Geometry of general climb model, showing an edge dislocation with segment CD in the glide plane and segment AC climbing over a particle. This is the same geometry as in Ref. [3], except for the cylindrical shape of the particle.

particles, which is too complex to be introduced in the present model. By symmetry of the elastic forces, only a positive edge dislocation is considered. The glide plane of the dislocation intercepts the particle at height $\mathrm{h}$ above its center; the height of the dislocation segment, above its glide plane upon climb over the particle is $z_{0}$ and the unraveling distance is $x_{0}$ (Fig. 1). Interactions between individual dislocations or between segments of a dislocation are neglected. By symmetry, it is also sufficient to consider a dislocation segment $\mathrm{AD}$ of length $\lambda / 2$, where $\lambda=r[\sqrt{3 \pi / 4 f}-1.64]$ is the mean-square lattice particle spacing (with $f$ the volume fraction of particles) [4]. The segment AD consists of a straight dislocation segment AB close to the particle surface and a curved segment $B D$ in the matrix. The segment $C D$ is entirely contained in the glide plane, whereas the segments $\mathrm{AB}$ and $\mathrm{BC}$ climb out of the glide plane. Under the action of an applied shear stress, $\tau$, the dislocation line BD in the matrix lies on a cylinder of radius of curvature, $R$ :

$R=\frac{G b}{2 \tau}$,

where $G$ is the shear modulus and $b$ is the Burgers vector of the matrix. The segment $A B$ is placed at a standoff distance corresponding to the point where the glide forces due to modulus mismatch $\left(F_{\mathrm{g}}^{\mu}\right)$ and lattice mismatch $\left(F_{\mathrm{g}}^{\varepsilon}\right)$ balance the force due to the applied stress, given by:

$\tau b \frac{\lambda}{2}+F_{\mathrm{g}}^{\mu}+F_{\mathrm{g}}^{\varepsilon}=0$.
The standoff distance cannot be smaller than a distance set arbitrarily to $b / 2$.

The first effect considered is the elastic interaction due to the modulus mismatch effect, arising between the coherent particle and the segment AB (interaction with the segment $\mathrm{BD}$ is neglected). The elastic strain energy associated with a dislocation is altered by the presence of particles with a different shear modulus and the resulting interaction energy depends on the modulus mismatch and the size of the particle. In most precipitationstrengthened alloys, the precipitate is stiffer than the matrix, so the dislocation is repelled by the particles. Because no simple exact solution exists for spherical particles, the solution derived by Dundurs [5] for an infinitely long cylindrical particle interacting with an straight edge dislocation is used to describe the modulus mismatch effect. The interaction energy $E$ for a unit length of dislocation is given by:

$$
\begin{aligned}
E= & \frac{G b^{2}}{\pi\left(k_{\mathrm{m}}+1\right)\left(1-\beta^{2}\right)}\left[\left(\alpha+\beta^{2}\right)\right. \\
& \times \log \left(\frac{y^{2}+(z+h)^{2}}{y^{2}+(z+h)^{2}-r^{2}}\right)+\left[\alpha+\beta^{2}-2(1+\alpha) \beta\right] \\
& \times \frac{r^{2} y^{2}}{\left(y^{2}+(z+h)^{2}\right)^{2}}-\frac{1}{2}(1+\alpha) \\
& \left.\times\left[1-\beta-\frac{(1-\alpha)(1+\beta)}{1+\alpha-2 \beta}\right] \frac{r^{2}(z+h)^{2}}{\left(y^{2}+(z+h)^{2}\right)^{2}}\right],
\end{aligned}
$$


where $y$ and $z+h$ give the dislocation position with respect to the center of the particle, and the parameters $\alpha$ and $\beta$ are given by:

$\alpha=\frac{\Gamma\left(k_{\mathrm{m}}+1\right)-\left(k_{\mathrm{p}}+1\right)}{\Gamma\left(k_{\mathrm{m}}+1\right)+k_{\mathrm{p}}+1}$

$\beta=\frac{\Gamma\left(k_{\mathrm{m}}-1\right)-\left(k_{\mathrm{p}}-1\right)}{\Gamma\left(k_{\mathrm{m}}+1\right)+k_{\mathrm{p}}+1}$.

The modulus mismatch parameter is defined as $\Gamma=G_{\mathrm{m}} / G_{\mathrm{p}}$ and the Poisson parameter is $k_{\mathrm{m} / \mathrm{p}}=$ $3-4 v_{\mathrm{m} / \mathrm{p}}$, where $v$ is the Poisson's ratio, and the subscripts $m$ and $p$ refer to the matrix and particle, respectively. The glide and climb forces acting on the dislocation are then obtained by differentiating the energy expression (Eq. (3)):

$\left[F_{\mathrm{g}}^{\mu}, F_{\mathrm{c}}^{\mu}\right]=\left[-r\left(\frac{\partial E}{\partial y}\right),-r\left(\frac{\partial E}{\partial z}\right)\right]$.

The glide and climb forces are repulsive when $\Gamma<1$ and attractive when $\Gamma>1$. A repulsive glide force opposes the forward glide motion of the dislocation during particle bypass, while a repulsive climb force can help or hinder the climb bypass, as discussed later.

The second source of elastic interaction originates from the constrained lattice parameter mismatch $\varepsilon$ between the coherent particle and the matrix, which creates a stress field around the particle interacting with the dislocation [4]. The glide and climb forces acting on the dislocation segment $\mathrm{AB}$ are obtained using the Peach-Koehler equation for the strain field around a spherical coherent precipitate acting on an edge dislocation [4]:

$\left[F_{\mathrm{g}}^{\varepsilon}, F_{\mathrm{c}}^{\varepsilon}\right]=\left[2 G \varepsilon r^{3} b \frac{3 y z_{0}}{\left(y^{2}+z_{0}^{2}\right)^{5 / 2}} \frac{r}{2}, 2 G \varepsilon r^{3} b \frac{-2 y^{2}+z_{0}^{2}}{\left(y^{2}+z_{0}^{2}\right)^{5 / 2}} \frac{r}{2}\right]$.

As before, the elastic interaction between the particle and the segment BD is ignored.

Following Rösler and Arzt [3], the kinetics of the climb process is determined by considering the flux of vacancies required for the dislocation to climb over the particle. The glide time between particles is thus assumed to be negligible as com- pared to the climb time $t_{\mathrm{c}}$ for the dislocation to climb over or under the particle, and the strain rate $\dot{\varepsilon}$ for creep deformation is then given by the Orowan equation:

$\dot{\varepsilon}=\rho b \frac{1}{1-\tau / \tau_{\text {Or }}^{*}} \frac{\lambda}{t_{\mathrm{c}}}$.

To account for the fact that some particles are bypassed instantaneously by the Orowan looping mechanism, the Orowan equation (Eq. (7)) contains a correction factor $\left(1-\tau / \tau_{\mathrm{Or}}^{*}\right)^{-1}[6]$, where $\tau_{\mathrm{Or}}^{*}$ is the effective Orowan stress given below. In Eq. (7), the density of mobile dislocations, $\rho$, is estimated from [7]:

$\rho=\left(\frac{\tau}{G b}\right)^{2}$.

The dislocation climb bypass time is determined assuming a vacancy mechanism as [3]:

$t_{\mathrm{c}}=\int\left[\frac{\pi D_{\mathrm{v}} r}{k_{\mathrm{b}} T b} \frac{\left|\mu_{\mathrm{ABD}}\left(x_{0}, z_{0}\right)\right|}{\left|\mathrm{d} A_{\mathrm{ABD}} / \mathrm{d} y\right|}\right]^{-1} \mathrm{~d} y$,

where $D_{\mathrm{v}}$ is the self-diffusion coefficient, $k_{\mathrm{b}}$ is the Boltzmann constant and $T$ is the absolute temperature. The change in area below the dislocation line, $\mathrm{d} A_{\mathrm{ABD}}$, is calculated using the geometry of the climbing segment [3]. The sign of the chemical potential and the change in area depends on the direction of the dislocation movement: vacancies are emitted or annihilated at the dislocation length whether the dislocation movement is upwards or downwards. The equilibrium shape of the dislocation line $\left(x_{0}, z_{0}\right)$ is found by setting the chemical potential along the dislocation line to be constant. When $x_{0} \rightarrow(\lambda / 2)-r$, climb becomes restricted and a new set of equations, which are derived as above, must be considered, as explained in Ref. [3]. The chemical potential $\mu_{\mathrm{ABD}}$ along the dislocation line is set constant, i.e., $\mu_{\mathrm{ABD}}=\mu_{\mathrm{AB}}=\mu_{\mathrm{BD}}$. The chemical potential at the particle interface corresponds to the driving force for adding vacancies to the dislocation segment [3]:

$\mu_{\mathrm{AB}}=\frac{\left[\frac{G b^{2}}{2}\left(\frac{\partial l}{\partial y}\right)_{\mathrm{BD}}-\left(F_{\mathrm{g}}^{\mu}+F_{\mathrm{g}}^{\varepsilon}\right)-\left(F_{\mathrm{c}}^{\mu}+F_{\mathrm{c}}^{\varepsilon}\right) \frac{\mathrm{d} z}{\mathrm{~d} y}\right]}{\frac{1}{a_{\mathrm{v}}} \frac{\mathrm{d} A_{\mathrm{AB}}}{\mathrm{d} y}}$, 
where $a_{\mathrm{v}} \approx b^{2}$ is the cross sectional area of a vacancy. Following Rösler and Arzt [3], the line length increase, $\partial l / \partial y$, is calculated for a small displacement $\delta y$, with the condition that the area, $A_{\mathrm{BD}}$, under the unraveling segment $\mathrm{BD}$ remains constant.

The model is implemented numerically, the input being material constants, geometrical parameters and the normalized applied stress, $\tau / \tau_{\mathrm{Or}}^{*}$. The model output is the average dislocation velocity $\lambda / t_{\mathrm{c}}$ where the climbing time, $t_{\mathrm{c}}$, is obtained through Eq. (9). Forces, chemical potentials and dislocation geometry are calculated at every increment of the movement of the dislocation in the glide plane, $\delta y$.

Fig. 2 shows the average dislocation velocity at $300{ }^{\circ} \mathrm{C}$, calculated using the materials parameters listed in Table 1 for an $\mathrm{Al}-0.3 \mathrm{wt} . \% \mathrm{Sc}$ alloy consisting of an $\mathrm{Al}$ matrix containing coherent $\mathrm{Al}_{3} \mathrm{Sc}$ precipitates $5.9 \mathrm{~nm}$ in radius. Fig. 2 illustrates the different behavior of the dislocation depending on whether the glide plane is above or below the particle center (i.e., $h>0$ and $h<0$ ). For one half of the particle $(h>0)$, the sum of the work done by the elastic forces is positive, so that the dislocation is helped in its climb bypass motion. Once it

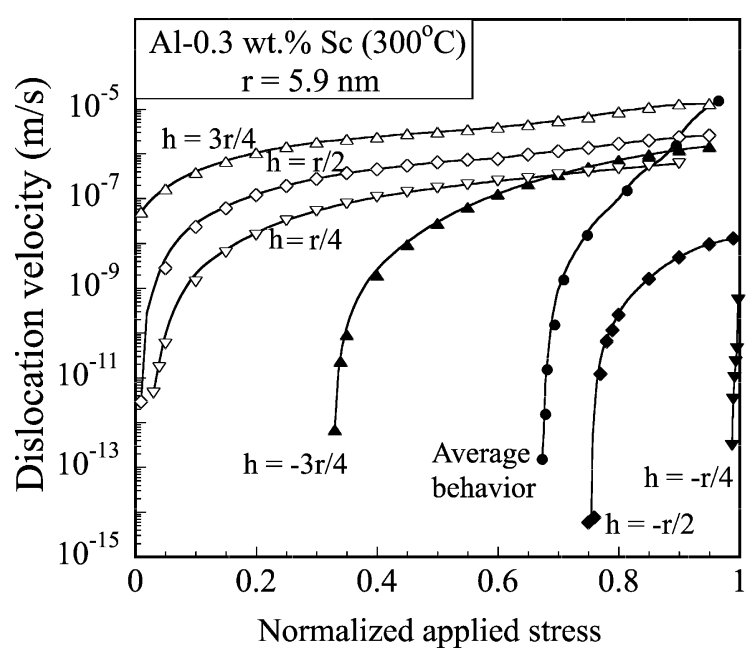

Fig. 2. Calculated average dislocation velocity $\lambda / t_{\mathrm{c}}$ at $300{ }^{\circ} \mathrm{C}$ as a function of normalized applied stress $\tau / \tau_{\mathrm{Or}}^{*}$ at different glide plane heights, $h$, for an $\mathrm{Al}-0.3 \mathrm{wt} . \% \mathrm{Sc}$ alloy with $5.9 \mathrm{~nm}$ radius precipitates. Parameters used in the model are listed in Table 1.
Table 1

Constants used in the calculations of strain rates for $\mathrm{Al}-$ 0.3 wt. $\%$ Sc at $300{ }^{\circ} \mathrm{C}$

\begin{tabular}{lll}
\hline & Values & References \\
\hline$G_{\mathrm{m}}(\mathrm{GPa})$ & 21.1 & {$[14]$} \\
$G_{\mathrm{p}}(\mathrm{GPa})$ & 63.0 & {$[15]$} \\
$v_{\mathrm{m}}$ & 0.34 & {$[15]$} \\
$v_{\mathrm{p}}$ & 0.2 & {$[15]$} \\
$b(\mathrm{~nm})$ & 0.286 & {$[14]$} \\
$f$ & 0.0075 & {$[10]$} \\
$\varepsilon$ & -0.0064 & {$[16]$} \\
$D_{\mathrm{v}}\left(\mathrm{m}^{2} / \mathrm{s}\right)$ & $2.6 \times 10^{-17}$ & {$[17]$} \\
\hline
\end{tabular}

reaches the top of the particle, the dislocation glides away from the particle, since the net glide force is positive (when $\Gamma<1$, as for Al-Sc alloys), so that the threshold stress is negligibly small. For the other half of the particle $(h<0)$, the total work done by the elastic forces is negative, so the dislocation is repelled from the particle interface and bypass can occur only when the applied stress is larger than a threshold value. An average curve is then obtained by taking an average on the stress at constant velocity over several heights $h$ in increment of $r / 16$ for the particle half with non-zero threshold stresses $(h<0$ in Fig. 2). However, the average velocity of a dislocation is twice that calculated above, because half the particles are intersected on average on their attractive half side $(h>0)$ and bypassed immediately. The average distance between repulsive obstacles then becomes $2 \lambda$, so the Orowan stress used to normalize the experimental and calculated creep data is:

$\tau_{\mathrm{Or}}^{*}=\frac{G b}{2 \lambda}$.

The asymmetry of the climb force due to lattice mismatch is responsible for the presence of a threshold stress. The modulus mismatch alone would not lead to significant threshold stress, because of the climb force that always helps the dislocation climb movement.

Finally, we note that the main effect of the elastic interactions is to modify the chemical potential of vacancies at the climbing section of the dislocation, so that both threshold stress and climb 
velocity are altered. This model predicts true threshold stresses for the dislocations, because the climb process cannot occur when the chemical potential for vacancies becomes positive. In that case, thermal activation, as used to modify detachment threshold stress for dislocations at the departure side of incoherent particles [9], does not come into play.

\section{Experimental procedures}

An $\mathrm{Al}-0.3 \mathrm{wt} . \% \mathrm{Sc}$ alloy was cast in graphite molds, homogenized at $648{ }^{\circ} \mathrm{C}$ for $24 \mathrm{~h}$, waterquenched to room-temperature and aged at $300^{\circ} \mathrm{C}$ for $5 \mathrm{~h}$. This aging treatment was followed by further aging at $400{ }^{\circ} \mathrm{C}$ for 3 and $10 \mathrm{~h}$ [10]. This resulted in a coarse-grained matrix containing a homogeneous distribution of coherent, spheroidal $\mathrm{Al}_{3} \mathrm{Sc}$ precipitates with a mean radius of $1.4 \pm 0.2$, $5.9 \pm 0.4$ and $9.6 \pm 0.9 \mathrm{~nm}$, respectively, as measured by image analysis of micrographs obtained by transmission electron microscopy [11]. Tensile creep testing was performed at $300{ }^{\circ} \mathrm{C}$ in air with an extensometer measuring tensile strain rate, $\dot{\varepsilon}$, under a uniaxial tensile stress $\sigma$, as reported in more detail in Ref. [12].

\section{Results and discussion}

Under creep conditions at $300{ }^{\circ} \mathrm{C}$, the $\mathrm{Al}-$ $0.3 \mathrm{wt} . \% \mathrm{Sc}$ alloys exhibit threshold stress behavior, as shown by the very high and variable apparent stress exponents in Fig. 3. The threshold stress, as found by plotting $\dot{\varepsilon}^{1 / n}$ vs. $\sigma$, increases monotonically from $0.04 \sigma_{\mathrm{Or}}^{*}$ to $0.57 \sigma_{\mathrm{Or}}^{*}$ for the three precipitate radii of $1.4,5.9$ and $9.6 \mathrm{~nm}$, where $\sigma_{\text {Or }}^{*}=M \tau_{\text {Or }}^{*}$, with $M=3.06$ the mean matrix orientation factor for aluminum [8]. In contrast, as shown in Fig. 3, the original general climb model predictions without elastic interactions (calculated as described above by setting $\varepsilon=0$ and $\Gamma=1$ and using a cylindrical particle) predicts threshold stresses of about $0.02 \sigma_{\mathrm{Or}}^{*}$, independent of the precipitate radius. This threshold stress is slightly lower than the value $0.06 \sigma_{\mathrm{Or}}^{*}$, predicted by Rösler and Arzt [3] for cubic particles. Models for inco-

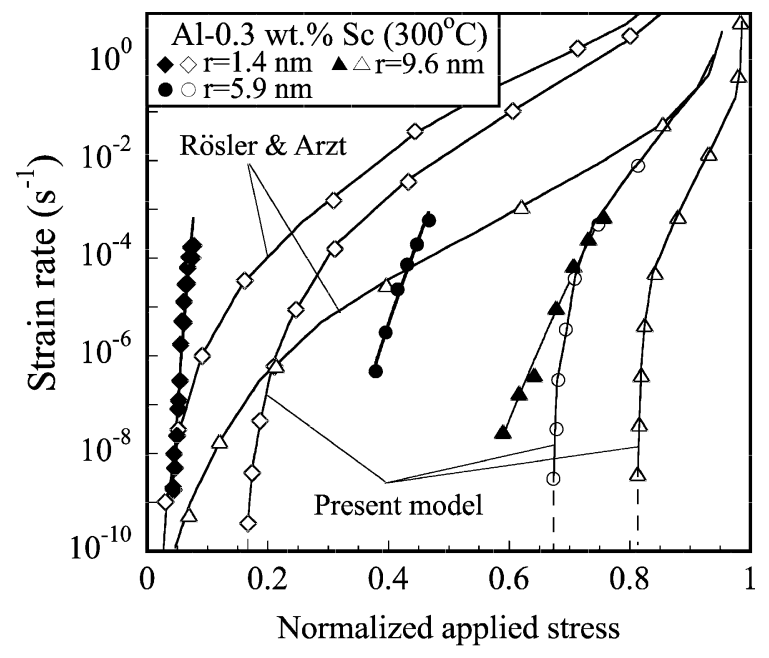

Fig. 3. Steady-state strain rate at $300{ }^{\circ} \mathrm{C}$ versus normalized applied stress $\sigma / \sigma_{\mathrm{Or}}^{*}$ for an $\mathrm{Al}-0.3 \mathrm{wt} . \% \mathrm{Sc}$ alloy containing coherent precipitates of various radii $r$. Filled and empty symbols are for measured and calculated data.

herent particles also give rise to a particle size dependence of the threshold stress [13]. However, these models based on attractive interactions at the departure side do not apply to the present case. As illustrated in Fig. 3 for precipitate radii of 1.4, 5.9 and $9.6 \mathrm{~nm}$, a small effect of particle size on strain rate is visible at stresses higher than the threshold value, because the concentration of vacancies requires for climb increases with increasing particle size. In contrast, taking into account the elastic interactions between the dislocations and the particles associated with the elastic modulus and lattice parameter mismatches $(\varepsilon=-0.0064$ and $\Gamma=0.33$, Table 1), the present, modified model predicts vastly different creep curves for different particles sizes (Fig. 3). The general trend of the creep data (i.e., increasing threshold stress with increasing precipitate radius) is successfully captured. Furthermore, the general shape of the stress-strain rate curves is reasonably well reproduced, considering the many assumptions in the model and the errors associated with the creep measurements and the values of the materials parameters. In particular, the strain rate in Eq. (7) is sensitive to the value of the dislocation density 
given by Eq. (8), which has been shown to be a poor approximation in dispersion-strengthened metals [3]. The calculated curves in Fig. 3 can thus be translated by many orders of magnitude along the $y$-axis, and their shape is more important than the exact value of the creep rate predicted. A major assumption is the trajectory of the climbing dislocation, taken as a circle. More thorough calculations would be required to take into account the forces acting on the dislocation and to determine the real trajectory by minimizing the dislocation energy. Another large source of error is the average stress calculated from the climb rates for various plane heights (Fig. 2). Finally, the alloy strength is likely overestimated by the choice of the mean square lattice spacing for the interparticle distance, $\lambda$, because of the random distribution of precipitates. Despite these qualifications, the present model provides a plausible explanation for the very high threshold stresses measured in $\mathrm{Al}-$ $0.3 \mathrm{wt} . \% \mathrm{Sc}$ alloys [10] and also reported in $\mathrm{Al}$ $0.2 \mathrm{wt} . \% \mathrm{Sc}$ in an earlier publication [12].

At high strain rates where the Orowan mechanism is operational, the alloy with the smallest particles is expected to have the highest creep resistance. On the other hand, the present model shows that at low strain rates where climb-bypass is active, large precipitate sizes are beneficial, provided that coherency is maintained. For an alloy where precipitates remain coherent to large radii, an optimum precipitate size should thus exist at intermediate radii, since the threshold stress is a trade-off between the Orowan stress (decreasing with precipitate size) and the repulsion due to modulus and lattice mismatches (increasing with precipitate size).

\section{Acknowledgements}

This research was supported by the United States Department of Energy, Basic Energy Sciences Division, under contract DE-FG0298ER45721. The authors thank Professors D.N. Seidman and G.B. Olson (Northwestern University), and Professor J. Rösler (Braunschweig University) for useful discussions.

\section{References}

[1] Cadek J. Creep in metallic materials. New York: Elsevier; 1998.

[2] Lagneborg R. Scripta Mater 1973;7:605.

[3] Rösler J, Arzt E. Acta Metall 1988;36:1043.

[4] Nembach E. Particle strengthening of metals and alloys. New York: John Wiley; 1997.

[5] Dundurs J. In: Mura T, editor. Mathematical theory of dislocations. New York: ASME; 1969. p. 70-115.

[6] Artz E, Ashby MF. Scripta Metall 1982;16:1285.

[7] Ashby MF. In: Kelly A, Nicholson RB, editors. Strengthening methods in crystals. Amsterdam: Elsevier; 1971. p. 137-91.

[8] Meyers MA, Chawla KK. Mechanical metallurgy: principles and applications. Englewood Cliffs, NJ: Paramus; 1984.

[9] Rösler J, Arzt E. Acta Metall Mater 1990;38:671.

[10] Marquis EA, Seidman DN, Dunand DC. Acta Mater, in press.

[11] Marquis EA, Seidman DN. Acta Mater 2001;49:1909.

[12] Fuller CB, Seidman DN, Dunand DC. Scripta Metall 1999;40:691.

[13] Mishra RS, Nandy TK. Philos Mag A 1993;69:1097.

[14] Frost HJ, Ashby MF. Deformation mechanism maps. Oxford: Pergamon Press; 1982.

[15] Hyland RW, Stiffler RC. Scripta Metall Mater 1991; 25:473.

[16] Harada Y, Dunand DC. unpublished results.

[17] Peterson NL. J Nucl Mater 1976;69-70:3. 